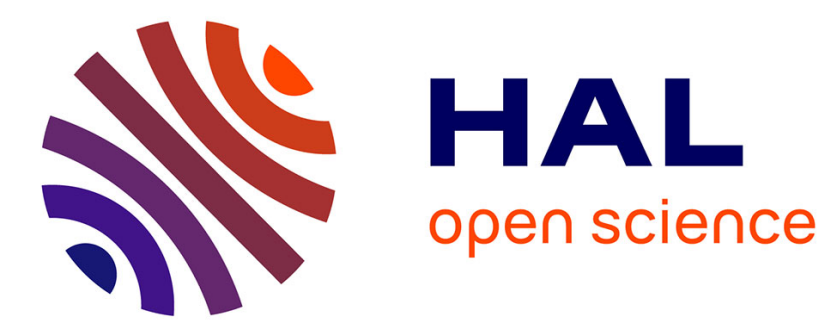

\title{
Deux millénaires de variations sur Krṣna: littératures, régions et religions
}

\author{
Denis Matringe
}

\section{To cite this version:}

Denis Matringe. Deux millénaires de variations sur Krṣna: littératures, régions et religions. Archives de Sciences Sociales des Religions, 2016, 176, pp.211-230. 10.4000/assr.28144 . halshs-01953484

\section{HAL Id: halshs-01953484 \\ https://shs.hal.science/halshs-01953484}

Submitted on 13 Dec 2018

HAL is a multi-disciplinary open access archive for the deposit and dissemination of scientific research documents, whether they are published or not. The documents may come from teaching and research institutions in France or abroad, or from public or private research centers.
L'archive ouverte pluridisciplinaire HAL, est destinée au dépôt et à la diffusion de documents scientifiques de niveau recherche, publiés ou non, émanant des établissements d'enseignement et de recherche français ou étrangers, des laboratoires publics ou privés. 
Archives de sciences sociales des religions

176 | octobre-décembre 2016

Bulletin Bibliographique

\title{
Deux millénaires de variations sur Krishna : littératures, régions et religions
}

\author{
Denis Matringe
}

\section{revues.org}

Édition électronique

URL : http://assr.revues.org/28144

DOI : $10.4000 /$ assr. 28144

ISSN : 1777-5825

\author{
Éditeur \\ Éditions de l'EHESS \\ Édition imprimée \\ Date de publication : 31 décembre 2016 \\ Pagination : 211-230 \\ ISSN : 0335-5985
}

Distribution électronique Cairn

CAIRN INFO

CHERCHER, REPÉRER, AVANCER.

Référence électronique

Denis Matringe, "Deux millénaires de variations sur Krishna : littératures, régions et religions », Archives de sciences sociales des religions [En ligne], 176 | octobre-décembre 2016, mis en ligne le 01 janvier 2019, consulté le 11 septembre 2017. URL : http://assr.revues.org/28144 ; DOI : 10.4000/ assr.28144 


\title{
Denis Matringe
}

\section{Deux millénaires de variations sur Krishna : littératures, régions et religions}

\author{
À propos de : \\ BRYANT Edwin F. (éd.), Krishna: A Sourcebook, New York, Oxford \\ University Press, 2007, 575 p., bibliographies. \\ COUTURE André et CHOJNACKI Christine, Krishna et ses métamorphoses \\ dans les traditions indiennes. Récits d'enfance autour $d u$ Harivamsha, \\ Paris, Presses de l'Université Paris-Sorbonne, 2014, 453 p., biblio- \\ graphie, index.
}

Au détour d'une rue, dans une ville européenne, un petit groupe de femmes et d'hommes vêtus d'habits safran pour les un-e-s - les célibataires - et blancs pour les autres chante, en s'accompagnant de petits tambours oblongs et de clochettes et en avançant avec des pas de danse, une litanie de trente-deux syllabes : "hare Krșna hare Krșna/Krșna Krșna hare harel/hare Rāma hare Rāmal Rāma Rāma hare harell ». Des Hare Krishna, comme on les appelle familièrement, mais, plus précisément, des membres de l'International Society for Krishna Consciousness (ISKCON), organisation religieuse hindoue fondée en 1966 à New York par le maître spirituel Abhaya-caraṇāravinda Bhakti-vedānta Svāmī Prabhupāda (1896-1977). Ces femmes et ces hommes, occidentaux pour la plupart, indiens pour certains, sont au nombre des hindous pratiquants les plus visibles en Europe ; ils prétendent se rattacher à un courant de dévotion à Krș̣na, le Gauṛiya Vaiṣnava Sampradāya fondé au Bengale - région autrefois appelée Gaurīya - par un mystique du nom de Caitanya (1486-1534). Pour eux, Krș̣na est " la suprême personnalité de Dieu », comme ils disent, et les textes qu'ils révèrent comme Écritures sont la Bhāgavad Gĩtā (c. II ${ }^{\mathrm{e}}$ siècle av. J.-C.) et, surtout, le Bhāgavata Purān (c. IX $\mathrm{IX}^{\mathrm{e}} \mathrm{X}^{\mathrm{e}}$ siècle) consacré aux manifestations terrestres ou avatāra (litt. " descente », en sanskrit) du dieu Vișṇu, avec un long livre X centré sur la geste de Krṣṇa, présenté comme l'Être suprême, supérieur à Viṣṇu lui-même.

Bhāgavad Gìtā et Bhāgavata Purāna font partie de l'immense océan de la littérature krṣṇaïte de l'Inde dont la production se poursuit depuis plus de deux millénaires, en diverses régions, diverses langues et divers contextes religieux. 
C'est à cette littérature qu'ont été consacrées, en 2007 et 2014, les deux remarquables anthologies objets de la présente note, qui se complètent parfaitement, mettant à la disposition des chercheurs et des étudiants des centaines de pages de sources traduites, introduites, présentées et annotées par certains des meilleurs spécialistes de l'univers krșṇaïte. Mais avant de scruter la constellation qu'elles dessinent et d'évoquer au passage d'autres travaux majeurs consacrés à Krṣ̣na ces dernières années avec lesquels ces deux anthologies sont en dialogue, il convient, pour les lecteurs non indianistes, de procéder à quelques rappels destinés à mieux situer les ensembles textuels qui seront évoqués et les groupes religieux au sein desquels ils sont nés ou ont été adoptés, voire adaptés, et la manière dont ils y fonctionnent.

\section{Krṣṇa un et multiple}

Nos deux anthologies rendent bien compte d'un fait incontournable : Kṛșṇa est un et multiple - et multiple au point d'être insaisissable dans toute sa diversité. Il peut être par exemple enfant turbulent ou grand roi, amant éminemment désirable se délectant dans les jeux érotiques ou cocher d'un prince à qui il révèle la Bhāgavad Gìtā ( "Chant du bienheureux »), guerrier impitoyable ou Dieu suprême, etc. Toutefois, au fil du temps, des morceaux épars du puzzle krș̣naïte ont été tant bien que mal rassemblés, avec pour fils conducteurs les Purāṇa et le Mahābhārata, qui inclut la Bhāgavad Gītā et un très important " complément " (khila, notion sur laquelle nous reviendrons) consacré à Krṣ̣na, le Harivamśa ( "la lignée de Hari »). Se sont ainsi dessinés les contours d'une biographie standard de Krș̣na dont se sont appropriés aujourd'hui un très grand nombre d'hindous. C'est cette biographie normée qui sert de trame, par exemple, aux dix grandes pages serrées consacrées à Krṣ̣na par l'écrivain, poète et savant kéralais Vettam Mani (1921-1987) dans sa Purānic Encyclopedia, parue d'abord en malayam en 1964 puis en traduction anglaise en 1975, à la série télévisée Śr Krṣna réalisée par Ramanand Sagar (1917-2005) en 1989 (Bombay, Sagar Entreprises) ou encore, en 1999, à la bande dessinée d'Anant Pai (1929-2011) racontant la vie de Krṣ̣na dans la célèbre collection Amar Chitra Katha ( immortelles histoires illustrées ») fondée par l'auteur en 1967.

Pour présenter ce Krș̣na à la fois standardisé et composite, nous suivrons ici la trame proposée par Guy Beck dans l'introduction de l'important livre qu'il a édité en 2005, Alternative Krishnas: Regional and Vernacular Variations on a Hindu Deity ${ }^{1}$. Krṣnna a censément séjourné sur terre pendant cent vingt ans environ, vers 3000 av. J.-C., au tournant de l'âge du monde qui est le nôtre, le kaliyuga, "âge de kali » ou "âge de fer ", quatrième et dernier de ceux qui constituent un cycle cosmique. Il l'a fait comme huitième des dix avatāra de Viṣnu

1. Beck, 2005. 
- lequel s'incarne chaque fois que le dharma est menacé parmi les humains -, alors que des démons des anciens temps avaient connu une nouvelle naissance sous la forme de rois mauvais. Dans cette manifestation, Krș̣na, le « bleu-noir », est accompagné d'un frère, Balarāma au teint clair, chacun étant né d'un cheveu de Viṣnu, respectivement un noir et un blanc.

Le pire des rois-démons est Kaṃsa, qui a détrôné son pieux père Ugrasena, souverain de la prestigieuse lignée royale des Yadāva remontant à Candra, « la Lune $»^{2}$. Kaṃsa fait régner la terreur au pays braj et dans sa capitale Mathura et c'est précisément dans sa famille, et dans cette ville, que naît Krṣṇa, huitième enfant de Devakī, sœur de Kaṃsa, et du mari de celle-ci, Vasudeva (c'est pourquoi Krọnna est aussi appelé Vāsudeva ou Vāsudeva Krọṇa). Or un songe a averti Kaṃsa qu'il périrait de la main du garçon huitième né de Devakī. Le roi fait donc enfermer sa sœur et Vasudeva et mettre à mort les six premiers fils qu'enfante Devakī. Le septième, Balarāma, est apparemment mort-né, alors qu'il a, en réalité, été transféré par une opération divine dans l'utérus de Rohinī, seconde épouse de Vasudeva. Quand enfin Krș̣na paraît, une semblable intervention permet un échange avec la fille que vient d'avoir, dans un village voisin, un couple aisé de vachers, Yaśodā et son époux Nanda.

Krṣṇa grandit, heureux et espiègle, chez ses parents adoptifs, mais Kaṃsa, ayant entendu parler de cet enfant miraculeux, envoie démons et monstres pour le tuer : en vain, car Krș̣na vient à bout de chacun de ceux-ci. Préadolescent, Krṣna accomplit divers autres miracles, comme soulever le mont Govardhana pour protéger les villageois d'une pluie diluvienne envoyée par Indra, roi des dieux, furieux que l'avatāra de Viṣnu ait demandé aux vachers de ne plus l'adorer et qu'il ait déclaré pouvoir assurer lui-même leur salut. Devenu jeune homme, Krṣ̣na séduit par sa personnalité, sa beauté et le chant de sa flûte filles et épouses des bouviers avec lesquelles il folâtre, danse à minuit, et dont il satisfait les désirs érotiques en se démultipliant.

Un jour, Kaṃsa invite Kṛṣṇa et Balarāma à un grand sacrifice dans le dessein de les tuer. Mais le messager envoyé pour transmettre l'invitation aux deux frères est un dévot de Krọṇa qui met ceux-ci en garde. Laissant les habitants du pays braj, et tout particulièrement les vachères, dans la douleur de la séparation, les deux frères se rendent à Mathura. Après avoir accompli plusieurs miracles, Kṛ̣ṇa et Balarāma exterminent Kaṃsa et huit de ses frères, et ils libèrent Vasudeva et Devakī, qui s'empressent de faire éduquer leurs deux fils dans tous les arts et toutes les sciences.

Mais le roi de Magadha, père de deux des épouses de Kamsa, attaque le pays braj. Krṣ̣ṇa emmène alors ses Yādava dans une nouvelle capitale qu'il fait surgir de terre, Dvārakā. Il épouse Rukmiṇī, fille du roi de Vidarbha, qui devient sa

2. L'autre grande lignée royale remonte à Sūrya, "le Soleil », est c'est d'elle qu'est issu Rāma, autre avatāra de Viṣnu et héros de la deuxième grande épopée sanskrite, le Rāmāyana. 
reine, ainsi que nombre d'autres femmes qui sont tombées amoureuses de lui ou lui ont été données par des rois et des princes reconnaissants - au nombre desquelles les seize mille filles de Nārakāsura.

Alors qu'il règne sur Dvārakā, Krọṇna s’émeut du sort de justes princes Yādava, les cinq Pāṇ̣ava, fils de Paṇdu et de ses épouses Kuntī et Mādrī. Les Pāṇdava ont été spoliés de leur royaume et de leurs biens par leurs cousins les Kaurava à l'occasion d'une partie de dés truquée. La guerre, en dépit des efforts de négociation de Krṣnạ, devient inéluctable entre les deux clans et leurs alliés. Elle éclate en effet et la grande épopée du Mahābhārata en raconte les dix-huit journées. Kṛsṇa s'engage au côté des Pāṇḍava et conduit le char de l'un des cinq frères, Arjuna. C'est le dialogue qu'il a avec ce dernier, soudain égaré à l'idée de devoir porter le fer contre ses cousins, qui forme la trame de la Bhāgavad Gìtāa, dans laquelle Krṣṇa prêche à Arjuna l'accomplissement du devoir dans le détachement des fruits qu'il peut rapporter et, se manifestant comme dieu suprême, révèle au jeune prince la voie du salut par l'amour de lui. Les Pāṇdava sont vainqueurs et les Yādava et Krṣ̣na retournent à Dvārakā. Mais Gāndhārī, la mère des Kaurava, leur a lancé une malédiction : Krṣna mourra dans la forêt, et les Yādava s'entredétruiront dans les trente-six années à venir. Il en va bien ainsi : les Yādava se tuent les uns les autres dans des querelles d'ivrognes et Krṣ̣na meurt des suites d'une blessure au pied que lui a infligée le chasseur Jara, le prenant pour une biche dans la forêt. Arjuna accomplit les rites funéraires de Krș̣na qui redevient Viṣnu dans le séjour céleste du Vaikuṇtha et est désormais adoré sous le nom de Nārāyaṇa. Il est rejoint en Viṣnu par Balarāma, qui a été à ses côtés jusqu'au bout, sauf pendant la grande guerre.

Dans l'immense champ du religieux indien, ce Krṣ̣na unifié dans ces reconstructions récentes et qui a fait l'objet de nombreuses interprétations savantes, lumineusement résumées par André Couture (professeur associé à l'Université de Québec) dans son livre L'enfance de Krșna (1991, p. 58-65), joue depuis quelque vingt-cinq siècles, sous une forme ou sous une autre, un rôle de premier plan dans la sphère de la dévotion théiste dite vaiṣnava (dévotion à Viṣnu), constituée de très nombreuses traditions sectaires ayant chacune leur fondateur, leurs Écritures et leurs rites ${ }^{3}$. De cette longue histoire atteste une masse considérable de documents. Si nos deux anthologies, comme nous allons le voir, proposent une excellente image des sources littéraires (je ne dis pas "écrites », car l'épigraphie et les monnaies en sont exclues), elles ne disent bien sûr rien des données archéologiques, pourtant fondamentales quand il s'agit de s'interroger sur les débuts de l'histoire complexe du dieu Krṣna : mais heureusement, il existe sur la question une somme magistrale de Charlotte Schmid (2010), directrice de recherche à l'École Française d'Extrême-Orient. Dans ce livre de sept cent cinquante

3. Pour une synthèse claire et informée sur ces traditions, voir Colas 2003 ; sur Viṣnu, voir la compréhensive mise au point récente d'André Couture dans la Brill Encyclopaedia Hinduism (Couture 2012). 
pages et comportant un cahier de soixante-trois planches, la chercheuse étudie les "premières représentations krș̣naïtes de la région de Mathura ", qui apparaissent au $\mathrm{II}^{\mathrm{e}}$ siècle av. J.-C. sur des monnaies et au $\mathrm{I}^{\mathrm{er}}$ siècle av. J.-C. dans la statuaire sous la forme d'une divinité à quatre bras. Pour ce faire, elle les croise avec les sources littéraires et épigraphiques, rappelant que les premières inscriptions qui attestent le culte de Vāsudeva Krṣṇa datent des environs de 150 av. J.-C. Ces représentations, dont Charlotte Schmid suit et analyse l'évolution, connaissent ensuite un grand essor à Mathura sous les empereurs Kuṣāna du ${ }^{\text {er }}$ au III ${ }^{\mathrm{e}}$ siècle ap. J.-C., à l'époque probable de la rédaction du Harivamśa et du développement la notion d'avatāra en liaison avec le renouveau de la figure du dieu védique Viṣnu ${ }^{4}$.

Dans l'introduction à l'anthologie qu'il a dirigée, Edwin F. Bryant revient pour sa part sur les premiers documents témoignant du culte de Kroṣna en Inde. Il rappelle que les premières attestations littéraires indubitables de ce culte se rencontrent d'une part dans des sources sanskrites des $\mathrm{V}^{\mathrm{e}}-\mathrm{IV}^{\mathrm{e}}$ siècles av. J.-C. - un dictionnaire étymologique, la fameuse grammaire du sanskrit de Pāṇini et un traité de dharma -, d'autre part dans des citations faites par des auteurs de l'antiquité classique des Indika perdus de Mégasthènes, qui fut ambassadeur séleucide à la cour de l'empereur indien Candragupta Maurya (r. c. 317-297 av. J.-C.), et enfin dans un texte de commentaire du canon bouddhique en pali, le Niddesa ( «Index»). D'autres se rencontrent dans des textes des III ${ }^{\mathrm{e}}$-II ${ }^{\mathrm{e}}$ siècles av. J.-C. : une Upanișad et le commentaire de la grammaire de Pāṇini par Patañjali (fl. II siècle av. J.-C.). Des environs de 110 avant l'ère chrétienne date une inscription figurant sur une colonne à Besnagar (Madhya Pradesh) dans laquelle Héliodore, ambassadeur du roi indo-grec Antialcidas à la cour du roi śunga Bhāgabhadra, se présente comme disciple de Krṣnna. Mention est faite ensuite par Bryant du Mahābhārata (assemblé pour l'essentiel entre le III siècle av. J.-C. et le III siècle ap. J.-C.), qui incorpore en son livre VI la Bhāgavad Gītā et le Harivamśa (ajouté vers le II siècle ap. J.-C.). Après avoir évoqué les premières représentations iconographiques de Krṣna, auxquelles est consacrée la somme de Charlotte Schmid, Bryant en vient à l'époque des empereurs gupta (IV ${ }^{\mathrm{e}}-\mathrm{VII}$ e siècles ap. J.-C.) - de dévots vaiṣnava - où le culte de Krṣṇa est répandu dans toute l'Inde du Nord et où les traces tant archéologiques que littéraires se multiplient : ainsi la naissance de Krọṇa est représentée dans le plus vieux temple connu de cette période, celui dit des dix avatāra à Deogarh, au sud de l'Uttar Pradesh actuel, et le Bālacarita ( "Les aventures du jeune [Krș̣na] ») de Bhāsa (fl. c. IV siècle) est la plus ancienne pièce de théâtre en sanskrit connue traitant de la geste de Krṣṇa.

4. Outre Schmid, 2010, on pourra également consulter Schmid, 2014, qui retrace l'apparition, dès le $\mathrm{VI}^{\mathrm{e}}$ siècle, de Krș̣̣a en joueur de flûte dans l'Inde méridionale et étudie le succès de cette image dans toute l'Inde. Signalons aussi deux importants articles consacrés à Kṛ̣ṇa dans Schmid et Francis 2014 : "Dharma, Yoga, and Viraha-Bhakti in Buddhacarita and Krșnacarita ", par Tracy Coleman (p. 31-61), et « The Infant Krșnaa in the Guruvāyūr Temple with Particular Reference to the Nārāyañiya of Nārāyanabhațța", par S. A. S. Sarma (p. 323-340). 
Mais surtout, c'est au temps de l'empire gupta que se développent des compilations brahmaniques appelées Purāṇa (litt. " ancien, vieux, antérieur » en sanskrit), dont le corpus s'enrichit ensuite jusqu'au XVI e siècle. Il s'agit de longs textes versifiés traitant de cosmogonie, des rois des temps anciens, des dieux et de leurs dévots, de théologies sectaires, de croyances concernant les pèlerinages, les lieux saints et les rites, et de pratiques yogi. La visée des auteurs ou des compilateurs de ces textes est d'évidence de légitimer dans une perspective brahmanique, à la lumière de l'orthodoxie védique, l'hindouisme théiste et sectaire des cultes de "dévotion" connu sous le nom de bhakti (litt. "partage » en sanskrit). Les dieux dont traitent principalement les Purāṇa sont Śiva et, surtout, Viṣnu, dont sont racontées en grand détail les incarnations terrestres. L'avatāra Kṛṇna apparaît ainsi dans le Viṣnn Purāna et le Padma Purāṇa, puis, plus tard, dans le Brahma Vaivarta Purāna, et bien sûr dans le Bhāgavata Purāna.

Ce dernier aurait été composé dans le Deccan vers le IX siècle (hypothèse dominante, mais débattue, nous y reviendrons), une partie de l'Inde où le Krṣnạisme avait été religion de cour à Kanchi et Maturai dans le Tamil Nadu dès le III è siècle et où la dévotion à Krṣ̣na était bien attestée dans la poésie en tamoul des Āḷār depuis le $\mathrm{VI}^{\mathrm{e}}$ siècle $^{5}$. Ce Purāṇa, avec son livre X consacré à Krṣnạa, allait devenir, dans toute l'Inde et jusqu'aujourd'hui, la matrice fondamentale de la littérature krṣṇaïte, lui « dont la beauté et la profondeur philosophique ont en quelques siècles conquis le cœur et l'intelligence des dévots de Krṣṇa " (Couture, in Couture et Chojnacki, 2014, p. 11).

Nous touchons ici au point nodal de nos deux anthologies pour ce qui concerne la constitution du corpus de cette littérature, et c'est Couture (ibid.) qui fait en quelque phrases clés la mise en perspective qui éclaire tout le paysage des textes krṣṇaïtes, notant que le Harivamśs - dont il est le meilleur spécialiste ${ }^{6}$ - est demeuré la version par excellence de l'histoire de Krṣna pendant environ un millénaire, se transformant et s'adaptant au fil des siècles en versions différentes selon les lieux et les milieux, avant que le Bhāgavata Purāna n'en devienne la version de référence. Dans une note importante, Couture (ibid.) précise en outre qu'une filiation peut être établie entre les deux textes, puisqu'il paraît " presque certain » d'une part que le Bhāgavata Purāna présuppose les hymnes en tamoul des Âlvār - saints poètes du sud de l'Inde ( $\mathrm{VI}^{\mathrm{e}}-\mathrm{IX}^{\mathrm{e}}$ siècles) dont les compositions

5. Contre une tradition savante bien établie (voir notamment Filliozat, 1962 et Hardy, 1983), Edwin F. Bryant, dans un article de 2002, argumente en faveur d'une composition du Bhāgavata Purāna à l'époque gupta et dans le nord de l'Inde, tandis qu'avec d'autres spécialistes, André Couture, reprenant les démonstrations de Jean Filliozat et Friedhelm Hardy, maintient que le Bhāgavata Purāna est un texte "qui a vraisemblablement été composé dans le sud de l'Inde vers la fin du premier millénaire de l'ère chrétienne " (Couture et Chojnacki, 2014, p. 11).

6. Outre l'ouvrage déjà cité (Couture, 1991) et son autre traduction introduite et annotée d'un passage du Harivamśa (Couture, 2007), on trouvera tout un ensemble d'études d'André Couture indispensables à la compréhension de ce grand poème réunies dans deux volumes sous le titre général Krṣna in the Harivariśa (Couture, 2015 et à paraître). 
témoignant d'une expérience personnelle de Dieu eurent une influence considérable sur le développement de la bhakti vaișṇava -, et d'autre part que ces poèmes semblent s'inspirer des version méridionales du Harivamśa - autant d'arguments qui vont dans le sens de l'hypothèse d'une origine méridionale du Bhāgavata Purāna).

Le tableau de la littérature krṣṇaïte peut à présent être complété. C’est donc comme figure centrale et mystérieuse du Mahābharata que Krșṇa fait sa véritable et majestueuse entrée dans la littérature sanskrite, à la fois comme héros et allié des Pāṇ̣ava dans leur lutte contre leurs cousins les Kaurava et comme incarnation de Viṣnu. C'est quasi concomitamment dans le Harivamśa qu'apparaît le premier récit développé et complet de l'enfance de Krș̣na, de sa vie d'adulte et de sa mort, qui inclut une peinture détaillée des dix années passées par l'enfant divin dans " une station forestière gérée quotidiennement par des bouviers » (ibid.). Autour du Harivamśs gravitent, du $\mathrm{II}^{\mathrm{e}}$ au XII ${ }^{\mathrm{e}}$ siècle, tout un ensemble d'écrits hindous, bouddhistes et jains : Couture en propose un tableau synthétique (ibid., p. 19). Mais dès le $\mathrm{X}^{\mathrm{e}}$ siècle, le Bhāgavata Purāna prend le relais et devient rapidement la source par excellence de la littérature krș̣naïte dans les diverses régions de l'Inde, en sanskrit et dans les langues dites vernaculaires.

Cet arbre généalogique littéraire, ce sont les travaux de Couture qui ont permis de le dresser. Krishna et ses métamorphoses non seulement complète, mais éclaire et met en perspective Krishna: A Source Book, ouvrage dans lequel n'est consacré au Harivamísa qu'un modeste chapitre III, "The Harivamsa: The Dynasty of Krishna ", par Ekkehard Loren (spécialiste des commentaires du Bhāgavata Purāna). Ce chapitre fonctionne comme une parenthèse dans l'anthologie dirigée par Bryant, puisque tout le reste du volume déploie la descendance du Bhāgavata Purāna.

L'ensemble formé par nos deux anthologies, dont l'articulation est - on l'espère - désormais plus claire, forme non seulement une mine en termes de sources, mais aussi un trésor pour la recherche en sciences sociales des religions : nous allons à présent, en passant de la première à la seconde avant de retourner à la première, tenter de montrer en quoi.

\section{Krọṇa dans le Mahābhārata}

Commençons donc avec Krishna: A Source Book, qui compte quatre parties : «Classical Source Material» (chapitres 1 à 4, p. 21-136), "Regional Literary Expressions " (chapitres 5 à 12, p. 136-306), "Philosophy and Theology » (chapitres 13 à 18, p. 307-474), et "Hagiography and Praxis" (chapitres 19 à 22, p. 475-575). Le Mahābhārata, auquel est consacré le premier chapitre de la première partie de cette anthologie (nous allons y venir), est le premier texte - et quel texte, avec plus de 200000 vers ! - à brosser un portrait de Krṣ̣na dans toute la complexité de sa double nature divine et humaine, faisant de lui un 
personnage central et, à l'arrière-plan, un moteur de l'action dans les livres $\mathrm{V}$ à XI de cette épopée qui en compte dix-huit. Ce grand poème, le lecteur occidental peut désormais le fréquenter dans une traduction anglaise intégrale et d'accès facile due à l'économiste indien Bibek Debroy et fondée sur l'édition critique de référence dite "de Poona "; il dispose aussi d'un guide de lecture d'une grande originalité réalisé par celle qui était l'une des meilleures spécialistes de cette épopée, Madeleine Biardeau (1922-2010), ancienne directrice d'études à l'École pratique des hautes études : deux remarquables volumes publiés par les éditions du Seuil avec un système de présentation distinguant soigneusement sur plus de deux mille deux cents pages le résumé serré du texte des analyses détaillées de la chercheuse ${ }^{7}$.

Des analyses de Madeleine Biardeau, Alf Hiltebeitel tient le plus grand compte dans "Krishna in the Mahabharata: The Death of Karna ", premier chapitre de la première partie de Krishna: A Source Book. Alf Hitebeitel y introduit, contextualise, traduit et commente l'épisode de l'épopée où est racontée la mort du héros tragique Karṇa, demi-frère aîné des Pāṇḍava combattant au côté des Kaurava. Krș̣na manœuvre de manière à assurer la victoire du Pāṇḍava Arjuna, dont il est le cocher et auquel il se révèle comme dieu suprême dans la Bhāgavad Gìtā : Karṇa n'est en effet vaincu qu'en raison d'une fatalité qui le perd, notamment quand son char de combat s'embourbe et s'immobilise - ce qui, du point de vue du dharma des guerriers, devrait faire cesser le combat contre lui. Mais comme le souligne Bryant, le même Krṣṇa qui doit permettre l'écrasement d'armées créant sur terre un grand désordre est aussi celui qui n'hésite pas à briser les codes du dharma pour sauver son dévot (bhakta) : la divinité de Krọṇ̣a se révèle être ici comme en d'autres passages un élément essentiel pour la compréhension de l'épopée. Cette divinité est au cœur de la magistrale étude consacrée par Alf Hiltebeitel à Krṣṇa dans le Mahābhārata (Hiltebeitel, 1976), dans laquelle l'auteur, prolongeant les recherches de George Dumézil dans Mythe et épopée (Dumézil, 1986, p. 236-265), montre que son héros, héritier de la mythologie sacrificielle indo-européenne puis védique et brahmanique, transmute, dans le grand sacrifice de la bataille, les forces du dharma en manifestation de la grâce divine dans un univers désormais caractérisé par la bhakti.

Rappelons ici pour l'historien des religions que cette vision diachronique a été amicalement critiquée par Madeleine Biardeau, qui avait du Mahābhārata une approche essentiellement structuraliste et synchronique. La chercheuse construit en effet la grande épopée d'une part comme un dispositif poétique destiné faire $\mathrm{du}$ Veda une manière d'herméneutique visant à donner expression à la nouvelle forme religieuse qu'est la bhakti, d'autre part comme la mise en scène d'une crise universelle rendant nécessaire la "descente » comme avatāra du dieu suprême

7. Voir dans la bibliographie Mahābhārata, 1933-1972, Debroy, 2010 et 2014, Biardeau, 2002. 
- crise dont le roi brahmanique sort fortifié, légitimé et assuré de la pérennité dans l'infinie succession des cycles temporels - et enfin et surtout comme une riposte brahmanique aux prétentions religieuses et politiques du bouddhisme qui se diffusait largement en Inde à l'époque de la composition des épopées et auquel s'était converti le grand roi Aśoka - thèse qui a fait l'objet de bien des controverses ${ }^{8}$.

La voie de salut qu'est la bhakti, qui semble déjà attestée dans une Upaniṣad plutôt liée à Śiva (Śvetāśvatara Upaniṣad), s'exprime avec force et beauté dans le célébrissime passage du Mahābhārata qu'est la Bhāgavad Gìtā, inlassablement commentée et traduite, en Inde comme ailleurs dans le monde ${ }^{9}$. Il consiste, nous l'avons vu, dans le dialogue, à la veille de grande bataille, entre le Pāṇdava Arjuna et son cocher Krșna. Ce dernier, comme le rappelle Robert N. Minor dans le chapitre II de Krishna: A Source Book, "Krishna in the Bhāgavad Gïta» (p. 7794), convainc Arjuna qu'il doit participer au combat bien que des membres de sa famille et de vénérés guru se trouvent dans le camp adverse. Après d'autres, Robert N. Minor voit la Gĩtā comme une interpolation de sept cents vers introduite vers 150 av. J.-C. à ce moment clé de l'épopée par un dévot de Krṣ̣na vénérant les Upanișad. Il montre dans son introduction comment la révélation de la dévotion à Krṣṇa émerge peu à peu de la scène de la préparation au combat, insistant sur le fait que dans la Gìtā, « la bhakti [...] n'a pas encore l'expression passionnée qui sera la sienne plus tard » (p. 78), en proposant en traduction des extraits précisément centrés sur ladite $b h a k t i^{10}$.

\section{Le Krș̣na du Harivamśa chez les hindous, les bouddhistes et les jains}

Pour la suite et dans la perspective qui est la nôtre ici, la contribution d'Ekkehard Lorenz sur le Harivamśa dans l'anthologie de Bryant (p. 95-109) doit renvoyer le lecteur vers Krishna et ses métamorphoses. Ce livre de Couture et Christine Chojnacki (professeure de sanskrit à l'université de Lyon III) est important pour l'historien des religions pour deux raisons principales. La première a déjà été évoquée : il y est rappelé par Couture le rôle matriciel du Harivamśa dans le développement de la littérature consacrée à Krṣṇa avant l'apparition du Bhāgavata Purāna. La seconde concerne les circulations textuelles entre religions en Inde : Couture et Chojnacki mettent en évidence - et il s'agit là d'une avancée considérable sur le front de la construction du savoir indianiste - que non seulement le Harivamśa a inspiré, au sein de la tradition hindoue, des textes introduisant des variantes de diverse nature dans l'histoire

8. Pour un aperçu synthétique, voir Biardeau, 2002, I, p. 20-24.

9. Pour le texte sanskrit et une traduction française de la Śvetāśvatara Upanișad, voir Silburn, 1948.

10. Rappelons que deux remarquables traductions françaises de la Bhagavad Gìtā ont été publiées au cours de ces dix dernières années (Ballanfat, 2007 et Hulin, 2010). 
de Krșna, mais aussi que, dans ce que Couture appelle «le monde fluide » de l'Inde (Couture et Chojnacki, 2014, p. 17), des moines bouddhistes et des maîtres jaina l'ont remanié « pour le plier aux exigences de leur [...] tradition et le rendre acceptable aux yeux de leurs coreligionnaires [...]. Sans en avoir l'air, et par touches successives, bouddhistes et jaina ont littéralement démoli le Krṣ̣na des hindous et l'ont remplacé par d'autres Krṣnạ, inoffensifs à leurs yeux » (ibid.).

Pour fonder cette démonstration, une section de l'introduction et toute la première partie de Krishna et ses métamorphoses sont consacrées par Couture au Harivamśa hindou et aux textes qui lui sont apparentés, l'accent étant résolument mis sur les récits d'enfance. Avant un résumé de l'enfance de Krṣ̣na d'après les chapitres XXX à LXXVIII du Harivamśa, qui en compte cent trente-cinq dans la version de l'édition critique, Couture, dans son introduction, procède à plusieurs mises au point importantes. La première de celles-ci concerne l'auteur du texte, qui serait un Bhāgavata, comme se désignaient les dévots de Krṣṇa et Balarāma apparus en Inde de l'Ouest ${ }^{11}$. La deuxième traite de la date du Harivamśa, et Couture, à partir d'une analyse serrée du texte et d'une réflexion sur sa nature, en situe la composition au $\mathrm{II}^{\mathrm{e}}$ ou au $\mathrm{III}^{\mathrm{e}}$ siècle ap. J.-C. ${ }^{12}$. La troisième porte précisément sur la nature de khila ou complément du Mahābhārata qui caractérise le Harivamśa et elle jette un jour neuf sur la sémantique de ce terme ${ }^{13}$. Pour Couture, "en tant même que khila, le [Harivamśa] est pensé comme un complément nécessaire, une œuvre qui s'est ajoutée à une autre dans le but de fournir davantage de précisions sur un sujet ou des données désormais jugées indispensables. Ce terme définit ainsi le rapport d'un texte avec un autre non en termes de date, mais d'utilité sur le plan de la compréhension » (ibid., p. 13). Un khila peut donc avoir coexisté avec le texte qu'il complète et y avoir été joint plus tard : il vaut donc mieux éviter de parler d' " appendice ", ce mot ayant une connotation minorante.

Après son résumé des chapitres XXX à LXXVIII du Harivamía d'après l'édition critique, mais en tenant compte des variantes rejetées en appendice par celleci, Couture propose la traduction de cinq textes de la tradition hindoue consacrés à l'enfance de Krș̣na, chacun étant dûment présenté et annoté (ibid., p. 43-126) : le premier, tiré du Mahābhārata, est l'un des passages de l'épopée qui laissent à penser que l'attribution à Krṣṇa d'une enfance merveilleuse est une tradition ancienne; les deux suivants sont les chapitres LXV et XCVI du Harivamśa; le troisième provient de la version sud-indienne du Mahābhārata; le dernier consiste en un extrait de la Bhäratamañjarī, poème cachemirien en sanskrit de Ksemendra (fl. milieu du XI ${ }^{\mathrm{e}}$ siècle) qui résume l'ensemble du Mahābhārata

11. Pour un aperçu synthétique sur les Bhāgavata, voir Colas, 2003 : 230-233.

12. Pour étude détaillée à ce sujet, voir l'article d'André Couture «Possibility of Assigning a Date for the Harivamśa ", in Couture, 2015, p. 67-87.

13. Voir aussi, d'André Couture, "The Harivamísa: A Supplement to the Mahābhārata ", in Couture, 2015, p. 9-21. 
et comporte, sur le modèle de l'original, un khila donnant la substance du Harivamśa (le passage traduit est tiré de ce khila). Il ressort de ces textes qu'à l'intérieur même de l'hindouisme, selon les époques, les lieux, les groupes et les locuteurs, « on ne parle pas toujours de Krṣ̣na de la même façon » (ibid., p. 44).

$\mathrm{Si}$, selon le consensus universitaire occidental, on situe la mort du Buddha vers 410 av. J.-C., le bouddhisme a déjà plus d'un demi-millénaire d'existence à l'époque où est composé le Harivamśa et il s'est à ce point répandu que pour la période qui va de 200 av. J.-C. à 200 ap. J.-C., on trouve en Inde beaucoup plus de ruines bouddhiques que de vestiges de l'hindouisme et du jaïnisme réunis. L'Ordre reçoit de nombreuses donations des fidèles, en diverses régions, des rois lui font présent de revenus de villages entiers, et deux grands empereurs se sont convertis, Aśoka, déjà rencontré et sous lequel le bouddhisme s'est imposé à Sri Lanka, et le souverain kuṣāna Kaniska (acc. 78 ap. J.-C.). Comme déjà à deux reprises auparavant, sous l'un et l'autre de ces empereurs ont lieu de grands conciles, à l'occasion desquels s'affirme la division de l'Ordre en diverses écoles. D'entre celles-ci, l'une des plus anciennes et la plus fidèle à l'enseignement du Buddha est celle des Sthaviravādin (en pāli theravād $\bar{\imath}$, " fidèles à l'enseignement des anciens "), qui s'implante à Sri Lanka. Dès le premier siècle av. J.-C., les moines sthaviravādin entreprennent de codifier leurs Écritures remontant censément à l'enseignement du Buddha, tandis qu'en sont rédigés des commentaires. Tout un ensemble comportant Écritures, commentaires et textes paracanoniques est finalement transcrit en pāli (langue moyen-indo-aryenne issue du sanskrit) au $V^{e}$ siècle ap. J.-C., et sans doute aussi modifié et augmenté, pour finalement aboutir à un ensemble de trois "Corbeilles" (tri-pițaka) - ainsi nommées d'après les contenants dans lesquels étaient disposées les bandes de feuilles de palmier servant de support à l'écriture : le vinaya ("comportement»), les sutta ( sermons ») et l'abhidhamma ( "métaphysique»). L'ensemble le plus long est celui des sutta, divisé en cinq groupes (nikāya), dont le dernier, dit khuddaka ( «mineur »), comprend certains des textes les plus célèbres du bouddhisme ancien comme le Dhammapada ("Vers sur la Loi »), les Theragāthā et les Therīgatha ( « Stances des doyens et des doyennes »), enfin les Jätaka (recueil des "Naissances »). Ces derniers forment un ensemble de plus de cinq cents poèmes retraçant brièvement des contes et des légendes présentés par le Buddha, narrateur putatif, comme se rapportant à ses vies antérieures, et destinés à illustrer un point de son enseignement. Chaque passage versifié y est accompagné d'un commentaire en prose (Jātakațthavaṇnanā, ou « exposition du sens du Jātaka) ${ }^{14}$.

Né au sein de la matrice brahmanique, le bouddhisme a d'abord été une religion de convertis et il s'est développé en Inde dans l'environnement de la

14. Comment ne pas mentionner ici le livre aussi vivant et drôle qu'érudit et remarquablement écrit consacré aux Jātaka par le grand indianiste et bouddhologue Alfred Foucher (1865-1952), - dernier ouvrage de cet auteur paru après son décès (Foucher, 1955). 
culture hindoue, dont il a gardé nombre de traits. La deuxième partie de l'anthologie de Couture et Chojnacki est ainsi consacrée, par le premier de ces auteurs, à «Un Krishna bouddhique » (p. 129-161). Couture y offre la traduction introduite et annotée du Ghatajātaka (Jātaka no 454), consacré à Krṣnạ, et de son commentaire. Dans sa présentation érudite, Couture retrace tout d'abord l'histoire de l'étude des Jātaka en général et celle du Ghatajātaka en particulier. Il se penche ensuite sur les rapports entre les quinze stances de ce Jātaka et leur commentaire, qui posent des problèmes ardus, comme celui de la relation entre l'histoire contenue dans le commentaire et celle que semblent supposer les stances éminemment elliptiques. Chemin faisant, Couture avance deux thèses. D'une part, " ce que dit ce texte (stances et commentaire), c'est la façon dont les bouddhistes doivent se représenter cette divinité hindoue [Krșṇa] s'ils veulent être fidèles aux intentions du Buddha »; d'autre part, et précisément dans cette perspective, "ce que le commentaire offre de plus précieux, c'est une contextualisation bouddhique des stances qui passe par l'histoire de [Krș̣na] telle qu'elle aurait été racontée par le Buddha " (Couture et Chojnacki, 2014, 132). Ces deux thèses sont amplement démontrées dans l'analyse minutieuse du texte à laquelle procède ensuite l'auteur, et dans laquelle il s'attache notamment à caractériser la «transformation » (p. 147) subie par le récit du Harivamśa dans le Ghatajātaka et, surtout, son commentaire.

La méthodologie mise au point par Couture pour aborder le Jātaka est appliquée dans les deux derniers tiers du corps de l'ouvrage (p.165-376) à un ensemble de textes jaina : est ainsi documenté un autre aspect de la fluidité des cultures de l'Inde et des transferts entre univers religieux.

Les jaina font remonter leur religion à un maître ascétique un peu antérieur au Buddha, Vardhamāna, appelé par ses disciples Mahāvīra ( Grand Héros ») pour être devenu un jina ( "conquérant ", d'où le nom des jaina), un tīrthankara ("passeur de gué »). Voie de libération fondée sur une absolue non-violence (ahimsa) et un ascétisme extrême pouvant aller jusqu'au jeûne à mort, le jaïnisme s'est comme le bouddhisme développé grâce au patronage des laïcs et s'est graduellement répandu dans toute l'Inde - des communautés apparaissant dans le Deccan dès le IV ${ }^{\mathrm{e}}$ siècle - et plus encore que le bouddhisme, il s'est avéré une religion schismatique. Le premier schisme, et aussi le plus fondamental, se produit dès le $\mathrm{IV}^{\mathrm{e}}$ siècle av. J.-C. et donne naissance à deux sectes : les Digambara ( "vêtus de ciel "), ainsi appelés parce que leurs ascètes vont nus, et les Śvetāmbara ( "vêtus de blanc »), dont les ascètes sont vêtus de blanc. Les deux sectes, qui se sont encore subdivisées par la suite sur des questions telles que la licéité du culte des images, ont beaucoup en commun, comme la conception d'un univers éternel passant indéfiniment par des cycles d'existence qui se répètent et voient chacun apparaître une série de soixante-trois hommes exceptionnels se succédant à intervalle régulier. Elles ont par contre des Écritures différentes. On trouve ainsi, dans l'anthologie de Couture et Chojnacki, un ensemble de six 
extraits traduits provenant des deux sectes. Deux d'entre eux sont tirés des Écritures des Śvetāmbara et ont été composés en prose prakrit pour l'un et en vers sanskrit pour l'autre par un auteur du Gujarat, respectivement au ve et au $\mathrm{XII}^{\mathrm{e}}$ siècle. Les quatre autres extraits proviennent des Écritures des Digambara et ont été écrits aux IX et $\mathrm{X}^{\mathrm{e}}$ siècles en vers sanskrits pour deux d'entre eux (l'un au Gujarat, l'autre en Inde du Sud) et en vers apabhraṃśa pour les deux autres, dont la région d'origine n'est pas précisée ${ }^{15}$. Comme il en va dans le chapitre précédent pour le Ghatajātaka, la traduction de chacun de ces textes par Chojnacki est précédée d'une introduction qui en donne le contexte et accompagnée de notes. L'ensemble est présenté en vingt-sept pages denses par Couture, qui offre à propos de ce corpus réflexions et observations destinées à "saisir en quoi les auteurs jaina ont modifié complètement le portrait de Krṣṇa enfant généralement accessible aux hindous » (p. 167) pour l'intégrer à leur propre univers religieux, pour faire de lui l'un de leurs soixante-trois grands hommes et pour faire de son histoire, à force d'additions, de modifications, de coupure, de changements dans le cadre cosmologique et historique, d'invention de nouveaux épisodes, " une histoire parfaitement jaina " (p. 169).

Dans son analyse de ces textes, Couture s'attache donc à caractériser les modifications opérées par les maîtres jaina: ajouts destinés à combler des lacunes, abrégements, transformations (de l'histoire des naissances, par exemple, ou encore du rapport entre Mathura et la station bovine), réaménagements structurels concernant des personnages ou l'espace et le temps. Ce faisant, ces maîtres " ont transplanté [les] récits [issus du Harivamśa hindou] à l'intérieur de leur propre modèle de libération » et ils " se sont montrés capables de réécrire toute l'enfance de Krṣna avec l'autorité et la conviction de personnes qui considèrent désormais cet ensemble d'épisodes comme une partie importante de leur propre histoire » (p. 192).

Si nous suivons Couture, il convient, pour être complet sur la postérité du Harivamśs avant le grand rayonnement du Bhāgavata Purāna telle qu'elle apparaît dans nos deux anthologies, de mentionner ici le chapitre VII de Krishna: A Source Book consacré à la poésie des Ālvār, sous le titre "Weaving Garlands in Tamil: The Poetry of the Alvars ", par Vasudha Narayan (p. 187-204). Cet auteur souligne, dans son introduction, l'importance des poèmes des Âlvār pour la secte viṣnuite tamoule des Śrī Vaiṣṇava, elle insiste sur la signification sociale de ces poètes et leurs œuvres (choix de la langue tamoule, poètes appartenant à différentes castes, et parmi eux une femme). Elle remarque aussi que dans la poésie des Āḷār, Viṣnu importe plus que ses avatāra, et aussi que le Krọṣna régional s'appelle Kannan ("chéri ») et son amante préférée - qui est aussi sa

15. Prakrit et apabhraṃśa renvoient à des états des langues indo-aryennes intermédiaires, dans cet ordre chronologique, entre le sanskrit et les langues modernes telles que hindi, bengali, etc. 
cousine - Nappinnai, que sa légende comporte divers épisodes originaux, et que l'univers de référence en est entièrement tamoul. Mais selon Couture, dans son compte rendu de Krishna: A Source Book, nombre de ces traits se trouvent aussi dans les versions méridionales du Harivamiśa (Couture, 2008, 145). Tout comme certains des textes jaina traduits par Christine Chojnacki dans Krishna et ses métamorphoses, les poèmes des Āḷār témoignent de la diffusion du Harivamíá en Inde du Sud (nous retrouvons ici, indirectement, la question déjà évoquée à deux reprises de l'origine méridionale du Bhāgavata Purāna).

\section{Le fabuleux destin du Bhāgavata Purāṇā}

À l'époque où est écrit dans le Gujarat l'un desdits textes jaina, le Triṣaștiśalālāpurușacaritra ( "La geste des soixante-trois grands hommes ») du moine Śvetāmbara Hemacandra, entre 1160 et 1172, une nouvelle étoile d'une brillance extrême et appelée à voir se former autour d'elles un nombre considérable de planètes est déjà apparue au firmament des littératures indiennes (et mondiales) : le Bhāgavata Purāna déjà plusieurs fois évoqué, composé en sanskrit dans le sud de l'Inde vers la fin du premier millénaire ap. J.-C., selon l'hypothèse la plus communément admise, et avec lequel nous sommes ramenés à l'anthologie dirigée par Bryant. C’est ce dernier qui, précisément, dans Krishna: A Source Book, présente le dixième livre du Bhāgavata Purāna, consacré à la geste krṣṇaïte, et en propose plusieurs extraits en traduction ("Krishna in the Tenth Book of the Bhāgavata Purāna ", p. 111-136) : il est d'autant mieux placé pour le faire qu'il a publié une traduction complète dudit livre ainsi que des chapitres 1, 6 et 2931 du livre XI (Bryant, 2003) ${ }^{16}$. Dans son introduction, après avoir situé le Bhāgavata Purāna dans le corpus des Purāṇa et rappelé sa position ouverte quant à la date et à l'aire géographique de la composition du texte, Bryant en vient aux questions de théologie et insiste sur quatre points. Premièrement, le Bhāgavata Purāna affirme la prééminence Krṣṇa sur toutes les autres manifestations de tous les dieux et sur tous les dieux, Viṣnu compris : "Ces autres incarnations sont partielles, mais Krṣnạ, lui, est le Bienheureux »(I.3.28, cité p. 114). Deuxièmement, Krș̣na n'est pas un simple accès vers l'absolu impersonnel (le brahman des Upanișad) qui le subsumerait ; mais le brahman comporte un élément personnel éternel en l'être de Krṣṇa. Troisièmement, toutes les activités de ce dieu suprême sont un «jeu» $(l \bar{l} l \bar{a})$. Le terme est notamment employé quand l'enfant divin

16. Rappelons ici la magnifique traduction française de l'ensemble du Bhāgavata Purāna entreprise par l'immense savant que fut Eugène Burnouf (1801-1852), fondateur de la Société Asiatique et titulaire de la chaire de langue et de littérature sanskrites du Collège de France de 1832 à 1852, et poursuivie après le décès de ce maître, pour le tome cinq et dernier par EugèneLouis Hauvette Besnault (1820-1888), professeur de sanskrit à l'École Pratique des Hautes Études, puis, après la disparition de celui-ci, par Alfred Roussel (1849-1921), professeur de sanskrit à l'université de Fribourg (Burnouf, Hauvette Besnault, Roussel, 1840-1898). 
s'amuse dans les beaux paysages du Braj, comme on appelle la région de Mathura et Vrindavan, et être assez intimement associé à Krș̣na pour participer à sa $l i ̄ l a ̂$ est « la plus haute perfection possible de l'existence humaine» (p. 115). Le dernier point, lié au précédent, concerne la yogamāyā, le divin pouvoir d'illusion par lequel Krṣṇa dissimule sa véritable identité de dieu suprême aux participants à sa lîlā de manière à éviter que, pétrifiés de stupeur, ils cessent de voir en lui un enfant, un ami, un amant, et d'interagir avec lui. Au demeurant, le pouvoir de cette lîlā s'étend au-delà de sa pratique concrète car méditer sur elle est un processus de yoga, d' " union » avec l'enfant cosmique. D'une manière générale, entendre ou lire l'histoire de Krș̣̣a, chanter le dieu, se le remémorer, lui rendre un culte, répéter son nom, se dévouer entièrement à lui, lui offrir tous ses actes et ses pensées, toute cela est bhakti-yoga et, partant, voie de libération. Même les ennemis du dieu, du simple fait qu'ils prononcent son nom ou pensent à lui obtiennent le salut. Dans cette perspective, le Bhāgavata Purāna est non seulement un livre d'histoire sainte, mais fonctionne aussi et surtout comme un substitut de Krṣṇa après que celui-ci a physiquement quitté le monde, comme « une incarnation littéraire du dieu » (p. 118).

Les autres chapitres de Krishna: A Source Book déclinent en quelque sorte le fascinant héritage du Bhāgavata Purāna, et plutôt que les présenter un à un, nous allons tenter de voir quel intérêt, ensemble, ils présentent pour les sciences sociales des religions.

Le premier fait frappant est la diffusion de l'histoire de Krṣ̣na telle qu'elle apparaît dans le Bhāgavata Purāna, mais non sans nombreuses variations, à l'échelle du sous-continent indien et dans ses diverses langues : sont ainsi présentés et traduits dans Krishna: A Source Book des textes écrits dans les langues de l'Assam (6), de l'Orissa (5) et du Bengale (12 et 22) pour l'est de l'Inde, du pays Braj pour le nord $(9,19)$, du Rajasthan (10) et du Gujarat (11) pour l'ouest, du Maharashtra (8) et du Tamil Nadu (7) pour le sud ${ }^{17}$. Il est à remarquer que la grande langue « cosmopolite » qu'est le sanskrit - selon le mot si juste de l'éminent indianiste étatsunien Sheldon Pollock (2009) - a continué à servir de medium pour la littérature krṣṇaïte au nord $(13,16,17,21,22)$ et au sud $(14,15)$.

Le deuxième fait ressortant clairement de cette anthologie est l'image qu'elle donne du fait sectaire dans l'univers de la bhakti - lumineusement caractérisé par Catherine Clémentin-Ojha dans son entrée "Hindouisme » du Dictionnaire des faits religieux dirigé par Danièle Hervieu-Léger et Régine Azria, auquel j'emprunte ce qui suit dans ce paragraphe ${ }^{18}$. Sur le plan de vie sociale, ce fait est caractérisé par la prolifération de groupes de dévots - renonçants et, souvent

17. Les numéros entre parenthèses renvoient aux chapitres de l'anthologie. Pour une évaluation analytique desdits chapitres, on pourra se référer au compte rendu susmentionné de Krishna: A Source Book par André Couture (Couture, 2008).

18. Clémentin-Ojha, 2010, p. 492-493. 
aussi, maîtres de maison - rassemblés autour d'un maître, lui-même héritier d'une lignée spirituelle remontant à un ascète fondateur souvent mythique qui a censément reçu de Dieu (Śiva ou Viṣṇu, ou l'un des avatāra de ce dernier) ou de la Déesse une révélation, source d'Écritures, généralement en sanskrit, mais parfois aussi en langue vernaculaire. Ces sectes sont appelées sampradāya (litt. «transmission ») en sanskrit. Leur monde est censé ne pas tenir compte de l'organisation sociale en castes hiérarchisées, on y entre - en théorie - par choix personnel, et la libération y dépend non l'exécution des devoirs de caste et de rites prescrits, mais de la relation d'amour fusionnelle établie avec la divinité et de la grâce accordée par celle-ci.

Pour ce qui est de la littérature sectaire, elle ne se limite pas aux textes issus de la révélation, mais comporte d'autres types d'écrits tels qu'hymnes à la divinité, poèmes consacrés aux émotions typiques de l'univers de bhakti que sont la douleur de la séparation de Dieu et la joie de l'union à lui ou chantant les louanges du fondateur ou de maîtres de la lignée, pièces de théâtre, traités théologiques, codes de conduite et de prescriptions rituelles, catéchismes, hagiographies, lettres des maîtres, etc. Krishna: A Source Book offre ainsi tout un florilège de textes provenant de plusieurs sectes majeures de la sphère vaișnava, ou qu'elles se sont appropriés. William L. Smith (6), par exemple, présente une pièce de théâtre écrite en Assam au XVI e siècle par Śankaradeva (1449-1568), fondateur en Assam de la secte Eka Nāma Śaraṇa Dharma ( Le dharma du refuge de l'Unique Nom »). John Stratton Hawley (9) traduit des poèmes chantant la vie de Krṣ̣na attribués à Súrdās (fl. XVI e siècle) et incorporés à son héritage par l'importante secte du Pustimārga ( "Voie de la grâce ») fondée à Vrindavan par Vallabhācarya (1479-1531), - tandis qu'au chapitre 19, Richard Barz traduit d'un célèbre recueil hagiographique de la tradition du Pușțimārga (dite aussi vallabhite) datant XVII ${ }^{\mathrm{e}}$ siècle des passages consacrés à Kumbhandās (1468c. 1582), paysan parangon de parfaite bhakti, et qu'au chapitre 20, Paul Arney écrit à propos d'un manuel vallabhite composite (XVI ${ }^{\mathrm{e}}-\mathrm{XVII}{ }^{\mathrm{e}}$ siècle) de préceptes et de pratiques traitant essentiellement du culte des images et du service de dévotion $($ sevā), et mêlant sanskrit et hindi du Braj. La contribution de Deepak Sarma (15) porte sur des traités et commentaires en sanskrit de Madhva (fl. $2^{\mathrm{e}}$ moitié du XIII ${ }^{\mathrm{e}}$ siècle ?), un maître né à Udipi au Karnataka selon les hagiographies, fondateur du Dvaita Vedānta Samprādaya ( «Secte du Vedānta dualiste ») - secte habituelle appelée Madhvā Sampradāya - et passé du Vedānta non dualiste de Śankkara (fl. VIII siècle) à la bhakti krșnạaite ${ }^{19}$. Satyanarayana Dasa consacre le

19. Pour rappel, le Vedānta ( fin du Veda ») est une tradition complexe et objet d'une abondante littérature qui prend appui sur l'enseignement de certaines grandes Upanișad affirmant l'identité entre l'absolu universel (brahman) et l'àtman (le «soi » individuel). Toutes les écoles du Vedānta ne sont toutefois pas non dualistes : certaines professent un non-dualisme dit qualifié, selon lequel le soi et le monde ne sont pas complètement identiques au brahman mais en sont des " modalités " (prakāra), d'autres un non-dualisme dualiste, fondé sur l'identité dans la différence, et d'autres enfin un Vedānta dualiste, maintenant une distinction irréductible entre le monde et le brahman, son créateur et seigneur, identifié à Viṣṇu. 
chapitre 16 à six traités théologiques composés en sanskrit par Jīva Gosvāmī (c. 1486-1534), né au Bengale et établi à Vrindavan, l'un des six successeurs immédiats du maître bengali Caitanya rencontré au début de cette note comme fondateur du Gauṛiya Vaiṣnava Sampradāya auquel se rattachent les Hare Krishna - tandis qu'au chapitre 17 David L. Haberman traite d'un texte sanskrit d'esthétique de la dévotion à Krṣṇa écrit par Rúpa Gosvāmī (trad. 1489-1564), oncle de Jìva et un autre des six successeurs immédiats de Caitanya, et qu'au chapitre 2), Neal Delmonico présente et traduit du bengali des poèmes de cette tradition.

Diversité des sectes, des lieux, des époques : la bhakti est un phénomène complexe, dont l'histoire est aujourd'hui encore d'autant plus mal assurée que les recherches à son sujet, en Inde notamment, ont souvent été informées par des partis pris idéologiques. On pourra lire à ce sujet, dans ce volume, le compte rendu par Catherine Clémentin-Ojha du livre de John Stratton Hawley, A Storm of Songs (Hawley, 2015 et Clémentin-Ojha, 2016). Notons ici, concernant les lieux, que l'anthologie de Bryant rend bien compte d'un phénomène sur lequel insiste dans son livre John Stratton Hawley : la centralité qui devient celle du pays Braj, où est né Krș̣na, pour les sectes krṣṇaïtes à partir du XVI e siècle.

Krishna: A Source Book, en même temps qu'il donne une image du fait sectaire en Inde, est riche de renseignements sur les pratiques religieuses qui ont cours dans la bhakti krșṇaïte. L'un des rites principaux de ce courant religieux consiste dans le chant de bhajan, poèmes des saints poètes médiévaux destinés au chant dans les assemblées de dévots appelées satsang : tel est, par exemple, éminemment le cas des poèmes de Súrdās traduits par John Stratton Hawley au chapitre 9. Une autre pratique très courante parmi les bhakta, consiste à méditer sur les noms de dieu ou à les répéter inlassablement, en vertu de leur pouvoir purificateur et salvifique (22, Neal Delmonico). Très importants pour les bhakta sont aussi d'une part le culte des images (20, Paul Arney), qui non seulement permet par des paroles, des chants et des offrandes de manifester son amour à dieu, mais qui est aussi l'occasion de "voir » dieu et de recevoir son regard de grâce ou darśana, et d'autre part le pèlerinage à des lieux saints en rapport avec la mythologie, en l'occurrence krșnaïte, pour recevoir le darśana du dieu à travers une image préservée dans un temple éloigné (21, Paul H. Sherbow).

Les poèmes ne sont pas les seuls textes à jouer un rôle dans la vie d'une secte de bhakta. Presque toutes les contributions de la troisième partie de Krșna: A Source Book sont consacrées à d'anciens traités en sanskrit de philosophie ou de théologie en lien avec la dévotion à Krș̣na : de tels textes continuent à être étudiés, commentés et enseignés par les maîtres sectaires. Quant aux hagiographies (19), si elles sont de même utilisées dans l'enseignement pour proposer des modèles de vie parfaite et de cheminement sans faille vers la libération, elles sont aussi la source principale pour la rédaction d'innombrables livrets en langues locales vendus sur les marchés et aux abords des temples et qui proposent des résumés de la vie de grands saints krș̣naiites. 
Tout cet ensemble textuel présenté dans les parties II, III et IV de l'anthologie dirigée par Bryant donne une image certes partielle (malgré les 575 pages du livre), mais assurément riche et parlante de l'immense héritage du Bhāgavata Purāna, prolongeant ainsi l'histoire commencée dans le chapitre I et poursuivie dans Krishna et ses métamorphoses par Couture et Chojnacki. En manière de contrepoint à ce dernier ouvrage, on aurait pu imaginer, dans l'anthologie dirigée par Bryant, un chapitre sur le long poème narratif consacré à la geste de Krṣṇa et inspiré du Bhāgavata Purāna dans le deuxième livre sacré des sikhs, le Dasama Grantha, compilé en hindi du Braj à la fin du premier tiers du XVIII siècle à Amritsar dans le Panjab par un ancien disciple du dixième et dernier guru des sikhs, Gobind Singh (1666-1708). Le Krș̣na des sikhs serait ainsi venu s’ajouter à ceux respectivement des hindous, des bouddhistes et des jains.

Mais telles qu'elles sont, ces deux anthologies très riches, érudites, mettent à la disposition des chercheurs un impressionnant corpus de sources provenant de la littérature krṣṇaïte, certaines traduites pour la première fois dans une langue européenne. Ensemble, elles racontent l'histoire de la formation de ce corpus, l'articulation entre les textes fondateurs que furent le Mahābhārata et son khila ( “complément ») le Harivamśa d'une part, et le Bhāgavata Purāna d'autre part, et elles suivent la diffusion de ces textes matriciels, jusqu'au début du premier millénaire pour le premier et jusqu'à nos jours pour le second. Elles donnent aussi une image de la "fluidité » qui existe entre l'hindouisme et les religions nées en son sein et offrent diverses pistes permettant d'explorer le foisonnant univers du fait sectaire hindou. L'une et l'autre ont fait l'objet d'un grand soin éditorial. À la différence de Krishna: A Source Book qui n'a - hélas - pas d'index, Krishna et ses métamorphoses en a trois (hindouisme, bouddhisme, jainisme), avec référence aux textes cités, et tous les mots indiens y sont translittérés alors qu'ils ne le sont pas dans l'autre anthologie.

Denis MATRINGE

CNRS, Paris

Centre d'Études de l'Inde et de l'Asie du Sud (EHESS-CNRS)

dmatringe@gmail.com

\section{Bibliographie}

Ballanfat Marc (traduction, introduction et notes), 2007, La Bhāgavad Gītā, Paris, Flammarion, coll. " G. F. ».

Beck Guy (dir.), 2005, Alternative Krishnas: Regional and Vernacular Variations on a Hindu Deity, New York, State University of New York Press.

Biardeau Madeleine, 2002, La Mahābhārata : un récit fondateur du brahmanisme et son interprétation, Paris, Éditions du Seuil. 
Bryant Edwin F. (éd.), 2002, "The Date and Provenance of the Bhāgavata Purāna and the Vaikunțha Perumāl Temple ", Journal of Vaishnava Studies 11/1, p. 51-80.

- (traduction, introduction et notes), 2003, Krishna: The Beautiful Legend of God (Śrìmad Bhāgavata Purāna Book X), London, Penguin, coll. "Classics».

-, 2007, Krishna: A Sourcebook, New York, Oxford University Press.

Burnouf Eugène, Hauvette Besnault Eugène-Louis, Roussel Alfred, 1840-1898, Le Bhâgavata Purâna ou histoire poétique de Krĭchna, 5 tomes, Paris, Imprimerie Royale, réimpr. avec une introduction de Jean Filliozat, Paris, Adrien Maisonneuve, 1981.

Clémentin-OJHA, Catherine, 2010, " Hindouisme », in Hervieu-Léger D., Azria R. (dir.), Dictionnaire des faits religieux, Paris, Presses universitaires de France, p. 490-499.

-, 2016, compte rendu de Hawley John Stratton, 2015, A Storm of Songs. India and the Idea of the Bhakti Movement, Cambridge (Massachusetts), London (England), Harvard University Press, in Archives de Sciences Sociales des Religions, n 176.

Colas Gérard, 2003, "History of the Vaiṣnava Traditions: An Esquisse ", in Flood G. (dir.), The Blackwell Companion to Hinduism, London, Blackwell, p. 229-270.

Couture André, 1991, L'enfance de Krishna, Paris, Éditions du Cerf, réimpr. Paris, Éditions du Cerf ; Laval, Presses de l'Université Laval, 2012.

-, 2008, compte rendu de BRYANT Edwin F. (éd.), Krishna: A Sourcebook, New York, Oxford University Press, 2007, in Journal of the American Oriental Society 128/1, p. 144-147.

-, 2012, "Viṣnu », in Jacobsen K. A., Basu H., Malinar A., Narayanan V. (éds.), Brill's Encyclopedia of Hinduism.

Couture André, CHOJNACKI Christine, 2014, Krishna et ses métamorphoses dans les traditions indiennes. Récits d'enfance autour du Harivamsha, Paris, Presses de l'Université Paris Sorbonne.

Couture André, 2015, Krșna in the Harivariśa, vol. 1 : The Wonderful Play of a Cosmic Child, New Delhi, DK Printworld.

-, à paraître, Krṣna in the Harivamísa, vol. 2, New Delhi, DK Printworld.

Debroy Bibek (trad.), 2010-2014, The Mahabharata, 10 vol., New Delhi, Penguin.

DumÉzIL Georges, 1986, Mythe et épopée I : l'idéologie des trois fonctions dans les épopées des peuples indo-européens, Paris, Gallimard, $2^{\mathrm{e}}$ édition, réimpr. dans Mythe et épopée, I. II. III., Paris, Gallimard, coll. «Quarto ", 1995.

Filliozat Jean, 1962, "Les dates du Bhāgavatapurāna et du Bhāgavatamāhātmya ", in Bender E. (éd.), Indological Sudies in Honour of N. Brown, New Haven, The American Oriental Society, p. 70-77.

Foucher Alfred, 1955, Les vies antérieures du Bouddha, d'après les textes et les monuments de l'Inde, Paris, Presses Universitaires de France.

HARDy Friedhelm, 1983, Viraha-Bhakti. The Early History of Krșna Devotion in South India, Delhi, Oxford University Press.

Hawley John Stratton, 2015, A Storm of Songs. India and the Idea of the Bhakti Movement, Cambridge (MA) and London, Harvard University Press.

Hiltebeitel Alf, 1976, The Ritual of Battle: Krishna in the Mahābhārata, New York, State University of New York (repr. with a Foreword by Wendy Doniger, 1991).

Hulin Michel (traduction, avec Émile Senart, introduction et notes), 2010, La Bhāgavad Gìtā, suivie du commentaire de Śankara (extraits), Paris, Points, coll. "Sagesses ». 
MAHĀBHĀRATA The, 1933-1972, For the first time critically edited, éd. critique de V. S. Sukthakar, S. K. Belvalkar et P. L. Vaidya, avec la collaboration de P. V. Kane, R. D. Karmarkar, V. G. Paranjpe, V. P. Vaidya, S. K. De, F. Edgerton, R. N. Dandekar, et al., 27 vol., Poona, Bhandarkar Oriental Research Institute.

Mani Vettam, 1975, Purānic Encyclopaedia, traduit du malayalam par Rajaram MenON P., Atchutan Nayyar K. R., Kartha R. K., John P. I. and Menon P. N., Delhi, Motilal Banarsidass Publishers.

PAI Anant, 1999, Krishna, Bombay, India Book House.

Pollock Sheldon, 2009, The Language of the Gods in the World of Men: Sanskrit, Culture, and Power in Premodern India, Berkeley and Los Angeles, University of California Press.

SCHMID Charlotte, 2010, Le Don de voir. Premières représentations krishnaïtes de la région de Mathurā, Paris, École Française d'Extrême-Orient.

-, 2014, Sur le chemin de Krșna. La flûte et ses voies, Paris, École Française d'ExtrêmeOrient, coll. «Sequens ».

SCHMID Charlotte, FrancIs Emmanuel, 2014, The Archeology of Bhakti I. Mathurā and Maturai, Back and Forth, Pondichéry, Institut Français de Pondichéry et Paris, École Française d'Extrême-Orient, coll. «Indologie ».

SIlbURn Aliette (traduction, introduction et notes), 1948, Śvetāśvatara Upaniṣad, Paris, Adrien Maisonneuve, réimpr. 1992. 